\title{
Examination of Spinal Cord Tissue Architecture with Magnetic Resonance Diffusion Tensor Imaging
}

\author{
Stephan E. Maier \\ Department of Radiology, Brigham and Women's Hospital, Harvard Medical School, Boston, Massachusetts 02115
}

\begin{abstract}
Summary: Magnetic resonance diffusion tensor imaging yields images with detailed information about tissue water diffusion. Diffusion-weighted imaging of the human spinal cord requires dedicated magnetic resonance pulse sequences that minimize the effects of subject motion, distortions, and artifacts from lipids and CSF flow. These problems are accentuated by the anatomic properties of the spinal cord (i.e., a small crosssectional dimension and a location deep inside the body). The diffusion tensor (a simplified model for complex diffusion in structured tissues) can be estimated for each image pixel by measuring diffusion along a minimum of six independent directions. It can then be used to derive mean diffusivity, diffusion anisotropy, and the dominant orientation of the diffusion process. The observation that diffusion along nerve fibers is
\end{abstract}

much higher than across fibers, allows a noninvasive reconstruction of the spinal cord nerve fiber architecture. This includes not only the primary cranio-caudad running connections, but also secondary, transverse running collateral fibers. With fiber tracking, the pixel-based diffusion information can be integrated to obtain a three-dimensional view of axonal fiber connectivity between the spinal cord and different brain regions. The development and myelination during infancy and early childhood is reflected in a gradual decrease of mean diffusivity and increase in anisotropy. There are several diseases that lead to either local or general changes in spinal cord water diffusion. For therapy research, such changes can be studied noninvasively and repeatedly in animal models. Key Words: Spinal cord, white matter, gray matter, MRI, eigenvector, diffusion imaging.

\section{INTRODUCTION}

The spinal cord is an integral part of the nervous system and interconnects the brain with the peripheral nervous system. The majority of nerve fibers in the spinal cord are myelinated. They run longitudinally and exit at the spine nerve roots at different segment levels. The central gray matter portion of the spinal cord contains not only longitudinal fibers, but also a large number of nerve cells and neuropil, a dense mesh of dendrites. Collateral fibers, which run largely orthogonal to the longitudinal fibers, connect the central gray matter with the surrounding white matter zone. These collateral nerve fibers interconnect different areas and levels of the spinal cord and form part of many functional connections within the spinal cord, such as the spinothalamic tract and the deep spinal reflex circuit. This reflex circuit enables rapid muscle contraction without any brain intervention after an appropriate sensory stimulus (e.g., heat).

Address correspondence and reprint requests to: Stephan E. Maier, M.D., Ph.D., Radiology (MRI), Brigham and Women's Hospital, Harvard Medical School, 75 Francis Street, Boston, MA 02115. E-mail: stephan@bwh.harvard.edu.
In routine clinical MR imaging, most of the signal arises from water in tissue and the visualization of tissue water diffusion properties with dedicated MR diffusion imaging sequences permits the noninvasive assessment of tissue architecture. Although the spatial resolution of clinical MR systems is typically limited to approximately $1 \mathrm{~mm}$, motion probing magnetic field gradients permit the detection of molecular diffusion at microscopic dimensions. In clinical scanners, each gradient is typically applied for a duration of several tens of milliseconds, during which time the average water molecule in biological tissues may migrate $10 \mu \mathrm{m}$ or more in a random direction. The sensitivity of the pulse sequence to the diffusion is expressed by the $b$-factor. A tissue-specific diffusion constant $d$ can be determined by measuring the diffusion-related signal attenuation, which equals $\exp (-d b)$. To eliminate signal variations due to $\mathrm{T} 2$ decay and nonuniform proton density, at least two measurements (i.e., one with diffusion encoding gradients and one without) must be performed.

In tissues, unlike pure liquids, the presence of various tissue components (i.e., large molecules, intracellular organs, membranes, cell walls) has an impeding effect on 
water diffusion. The observed diffusion is isotropic if this restriction is direction independent. It is isotropic if this restriction enforces a preferred orientation of the water diffusion process that is largely uniform across the entire imaging voxel. The directional variability of restricted diffusion is usually quantified by the rotationally invariant fractional anisotropy (FA) index, ${ }^{1}$ which ranges from 0 (isotropic diffusion) to $100 \%$ (diffusion along one orientation only).

In the brain and spinal cord, diffusion anisotropy is attributed to the presence of densely packed axonal membranes. 2,3 The motion of tissue water across the axon membranes and myelin sheath that surround the axons is restricted. At the same time, water molecules are relatively free to move along the longitudinal direction of the axons. The capability of MR diffusion imaging to quantitatively probe diffusion along arbitrary directions combined with the concept of the diffusion tensor ${ }^{4}$ permits the accurate assessment of the preferred diffusion direction for each imaging voxel. With the diffusion tensor model, diffusion can be conceptualized and visualized with ellipsoids; the directions of the orthogonal ellipsoid main axes represent the diffusion tensor eigenvectors and their length represent the diffusion tensor eigenvalues $\lambda_{1}, \lambda_{2}$, and $\lambda_{3}$. The first eigenvector, with the highest diffusivity $\lambda_{1}$ points along the dominant diffusion direction, which coincides with the longitudinal direction of the nerve fiber bundles. ${ }^{5,6}$ The third eigenvector, with the lowest diffusivity $\lambda_{3}$, points along the direction of most restricted diffusion. In the absence of any other structures, such as crossing nerve fibers or supporting cells, the second and third eigenvector point in random directions perpendicular to the longitudinal axis of the fiber bundle.

\section{MR PULSE SEQUENCES FOR SPINAL CORD DIFFUSION IMAGING}

Magnetic resonance diffusion imaging is routinely performed in the brain during clinical or research examinations and suitable MR pulse sequences are available on most clinical MR systems. In the spinal cord, however, the application of standard pulse sequences for diffusion imaging results in inadequate image quality for several reasons: 1) the small cross-section of the spinal cord requires high-resolution imaging to obtain any meaningful anatomical information, and because the spinal cord runs deep inside the body, it is difficult to simultaneously achieve an acceptable signal-to-noise ratio and high spatial resolution; 2) The surrounding CSF is likely to confound the measurements due to partial volume effects, unless images are obtained at high resolution; 3) motion of the CSF and organs in the thorax and abdomen may lead to ghosting artifacts, unless single-shot diffusion imaging techniques are used; 4) the presence of nearby bony structures causes magnetic susceptibility variations ${ }^{7}$ that can not be compensated with magnetic field shimming, and although smaller than the susceptibility variations at air-tissue interfaces, they nevertheless may lead to serious image distortions; 5) chemical-shift artifacts arise from lipids in the vertebral bodies and other nearby structures. Fat signal suppression may not fully eliminate the chemical-shift artifacts, because it may be difficult to shim the magnetic field homogeneity over an extended length of the spinal cord.

The standard single-shot echo-planar diffusion imaging sequences $^{8}$ that acquire a complete diffusionweighted image after a single radio-frequency excitation, offer limited spatial resolution and signal-to-noise ratio. Moreover, these sequences are particularly sensitive to local magnetic field variations, chemical shift, and eddy currents. The resulting artifacts are more severe at higher magnetic field strengths. Multi-shot, diffusion-weighted, spin-echo sequences largely overcome these limitations, but suffer from severe motion artifacts. To some extent these motion artifacts can be mitigated by avoiding intervals of strong motion with a cardiac-gated acquisition ${ }^{9}$ and by monitoring and compensating motion with navigators. This technique was used in the first report of in vivo spinal cord diffusion images obtained in humans. ${ }^{10}$ Cardiac gating increases scan time and with the sequence described in this first report, $30 \mathrm{~min}$ or more are needed to acquire the minimal number of six diffusion encoding directions to compute the diffusion tensor. More recent studies with navigator correction have used interleaved echo-planar diffusion imaging ${ }^{9,11-15}$ and have achieved a considerably shorter scan time. All other approaches that have been suggested for spinal cord diffusion imaging use single-shot imaging methods and do not require cardiac gating. Standard single-shot echo-planar techniques, despite the tendency to produce images with the most severe distortion artifacts, have been used for spinal cord diffusion-weighted imaging. ${ }^{16}$ Voss et al. ${ }^{17}$ used two spinal cord image acquisitions with opposite phase encode gradient polarity to estimate and correct distortions. Various other modifications, which have been incorporated to decrease the susceptibility-related distortions and chemical shift artifacts, invariably reduce the signalto-noise ratio or scan speed. Fast spin-echo single-shot diffusion-weighted imaging ${ }^{18,19}$ uses refocusing pulses to obtain multiple signal echoes after a single radiofrequency excitation. In a comparative spinal cord diffusion imaging study, ${ }^{20}$ however, navigated spin-echo sequences were found to outperform fast spin-echo sequences. The human spinal cord data presented in this article was obtained with line scan diffusion imaging, ${ }^{21,22}$ a spin-echo technique that relies on the sequential single-shot acquisition of columns. Despite limited scan speed, rectangular images with diffusion-weighted images along six encoding axes can be scanned in as 


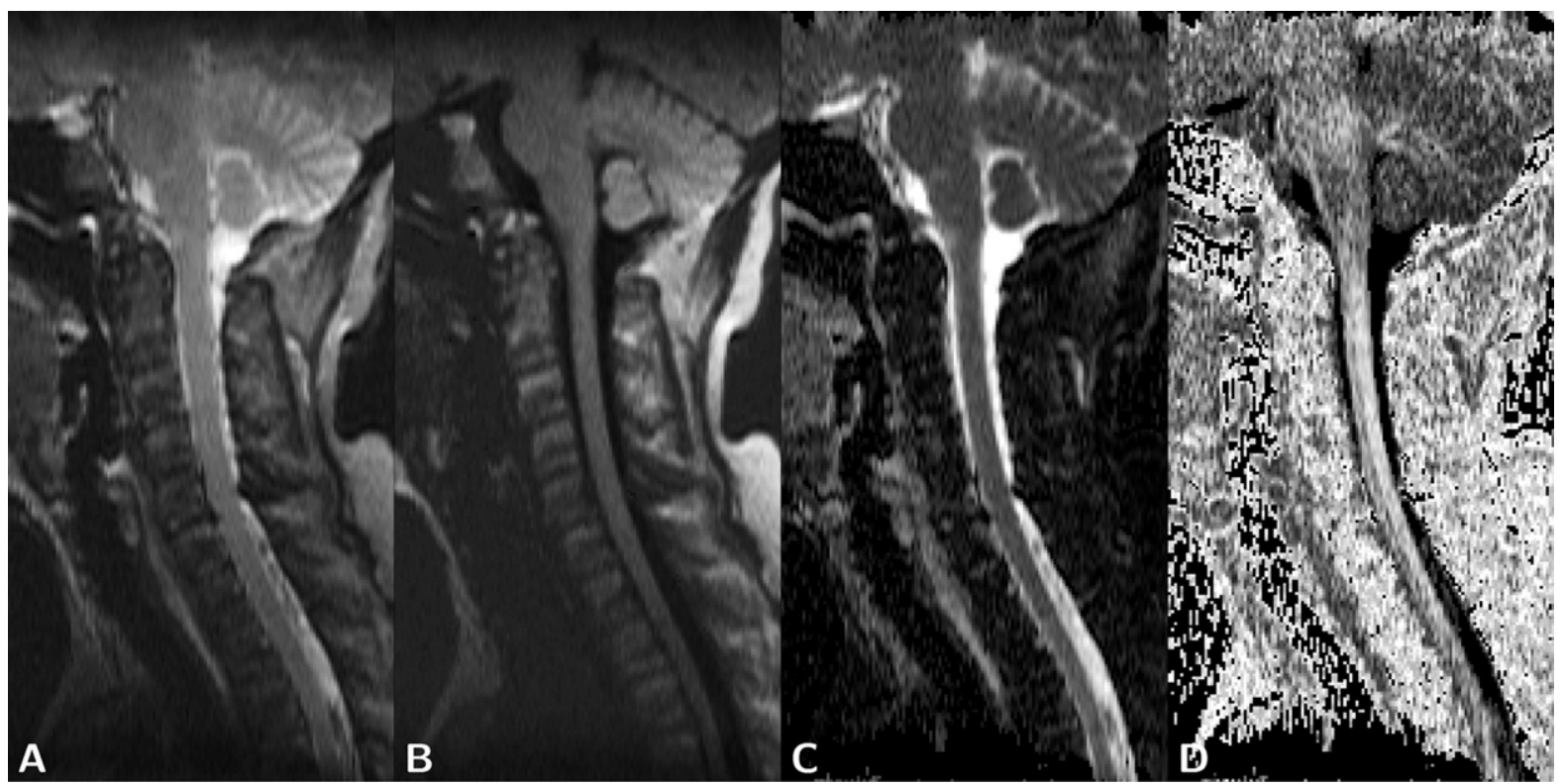

FIG. 1. Sagittal line scan diffusion tensor image data of the cervical spinal cord in a healthy 48-year-old female volunteer. A: T2-weighted image. B: Diffusion-weighted image reconstructed from image data along six diffusion encoding directions and extrapolated to $b=1000 \mathrm{~s} / \mathrm{mm} 2$. C: Trace diffusivity map. D: Fractional anisotropy (FA) map. (Note the band of slightly lower FA values in the ventral portion of the spinal cord.)

little as $25 \mathrm{~s}$ per section. ${ }^{23}$ Moreover, line scan diffusion imaging performs reliably at low, ${ }^{24}$ intermediate, ${ }^{25}$ and high-field strengths. Between the extremes of single column excitation and standard full field of view, singleshot imaging, there are methods that achieve artifact reduction through reduced field of view imaging ${ }^{26-28}$ or parallel imaging. ${ }^{29,30}$

Typical scan parameters for human spinal cord scans constitute an axial or sagittal scan plane, with a field of view of 200 to $300 \mathrm{~mm}$ that may be reduced along one direction, a spatial in-plane resolution of 1.0 to $2.0 \mathrm{~mm}$, and a slice thickness of 3 to $4 \mathrm{~mm}$. As for brain diffusion tensor scans, a b-factor between 750 and $1000 \mathrm{~s} / \mathrm{mm}^{2}$ is ideal. To obtain a rotationally invariant measure of tissue water diffusion (i.e., trace of the diffusion tensor), a minimum of three orthogonal diffusion encoding directions suffice, whereas for the complete diffusion tensor, a minimum of six noncollinear and noncoplanar encoding directions are required. For efficient coverage, phased-array receiver coils are preferred, but individual surface coils may permit improved resolution over a limited field of view. To maximize the MR signal, the echo time should be kept as short as possible, typically in a range between $60 \mathrm{~ms}$ (line scan diffusion imaging) and $100 \mathrm{~ms}$ (single-shot echo-planar imaging). The repetition time, on the other hand, should be as long as possible to avoid T1-weighting. If much longer scan times are acceptable, CSF signal suppression can be applied to reduce partial volume effects and obtain more accurate measurements of white matter diffusion. ${ }^{31}$ To improve spatial resolution and avoid overestimation of diffusion anisotropy in areas of low anisotropy, ${ }^{25,32}$ signal averaging may be necessary. In situations of an extremely low signal, the effect of magnitude signal averaging and the resulting Rician noise distribution must be considered and corrected. ${ }^{33}$ Measurements of the eigenvector direction also benefit from signal averaging, but unlike FA values, they are free of any bias. Instead of simple repeat

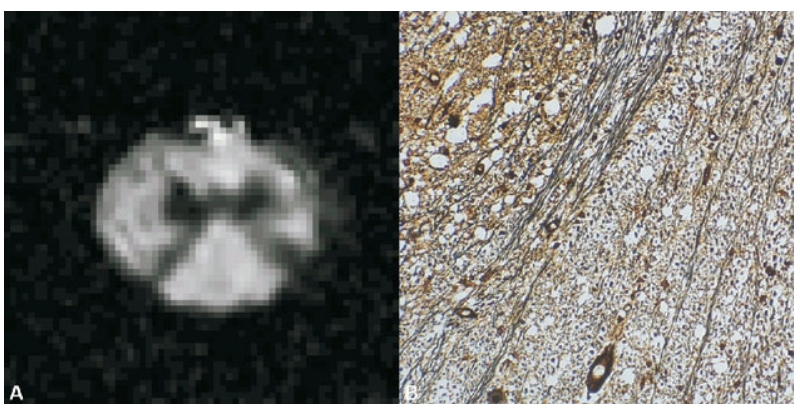

FIG. 2. Human cervical specimen from the C1-C2 level of a 34-year-old deceased female patient who had no known abnormality of the spinal cord. A: Fractional anisotropy (FA) map of an axial specimen section. Bottom of the image corresponds to dorsal. The FA map exhibits two well-characterized zones of different intensity. The central butterfly-shaped dark zone of low anisotropy corresponds to the central gray matter, whereas the surrounding bright zone of high anisotropy corresponds to the peripheral white matter. B: Typical microscopy view of an axial histology section of the same cervical spinal cord after staining according to Bielschowsky's method of silver impregnation. The upper left corner area shows a zone of gray matter, distinguishable by the brownish appearing neuropil. The adjacent white matter zone is dominated by axons, which appear as small black dots with a surrounding halo of dissolved myelin. Collateral axons, which run in-plane from the upper right corner to the lower left corner, are also visible. 
TABLE 1. Spinal Cord Diffusion Parameters

\begin{tabular}{lccc}
\hline & $\begin{array}{c}\text { Diffusivity } \\
\mu \mathrm{m}^{2} / \mathrm{ms}\end{array}$ & $\begin{array}{c}\text { FA } \\
\text { No Signal Averaging }\end{array}$ & $\begin{array}{c}\text { FA } \\
\text { 20 Signal Averages }\end{array}$ \\
\hline Spinal cord (sagittal), adults (age, 32-48 years) & $0.81 \pm 0.03[38]$ & $0.70 \pm 0.05[38]$ & \\
Spinal cord (axial), white matter, adults & $0.87 \pm 0.04[39]$ & $0.72 \pm 0.03[25]$ & $0.65 \pm 0.04[25]$ \\
Spinal cord (axial), gray matter, adults & $0.81 \pm 0.02[39]$ & $0.53 \pm 0.09[25]$ & $0.34 \pm 0.05[25]$ \\
Cortex, adults & & $0.20 \pm 0.03[25]$ & $0.09 \pm 0.01[25]$ \\
Spinal cord (axial), cranio-caudal, adults & $1.648 \pm 0.123[27]$ & \\
Spinal cord (axial), perpendicular, adults & $0.570 \pm 0.047[27]$ & \\
Spinal cord (sagittal), children (age, 2 months-18 years) & $0.96 \pm 0.05[23]$ & \\
Spinal cord (sagittal), infants (age, 28-40 weeks & $1.25 \pm 0.10[37]$ & \\
postmenstrual) & & \\
Cerebro-spinal fluid & $3.423 \pm 0.16[34]$ & \\
\hline
\end{tabular}

$\mathrm{FA}=$ fractional anisotropy.

measurements, it may be more useful to acquire more diffusion encoding directions.

\section{DIFFUSION PARAMETERS OF THE NORMAL SPINAL CORD}

Spinal cord diffusion image data of a healthy volunteer is presented in Figure 1. There is no evidence of severe image distortion, ghosting, or motion-related signal loss. On the diffusion-weighted image (FIG. 1B), the normal spinal cord exhibits uniform signal. The surrounding CSF appears dark, due to a strong diffusion-related signal loss. ${ }^{34}$ Accordingly, on the diffusion map (FIG. 1C), the high diffusivity of CSF is reflected by the bright appearance of the CSF, which contrasts with the much lower diffusivity of the spinal cord. On the FA map (FIG. 1D), much more noise is manifest. Nevertheless, a narrow longitudinal strip along the ventral side of the spinal cord with a lower anisotropy value than the rest of the spinal cord can be discerned. Most likely, it can be attributed to the gray matter core of the spinal cord. The difference in diffusion anisotropy between spinal cord gray and white matter is clearly visualized on the axial FA map of a human spinal cord specimen shown in Figure 2A. The corresponding histology of neighboring gray and white matter zones is presented in Figure 2B. In agreement with the theory of directionally dependent diffusion restriction by axons, ${ }^{2,3}$ the axon density in the high-anisotropy white matter zone appears much higher. Nevertheless, the presence of myelinated cranio-caudal and collateral nerve fibers in the spinal cord gray matter gives rise to a much higher FA value than is typically observed in the cortical gray matter. Manual ${ }^{35}$ and computer-aided ${ }^{36}$ analysis of spinal cord specimens demonstrate a good correlation between FA and axon density. In particular, it was found that the dorsal running tract of epicritical and proprioceptive sensation fibers is clearly visible as a triangular-shaped area of high amplitude in corresponding locations in both FA and axon density maps. ${ }^{36}$

Average diffusion and FA values of the normal spinal cord are listed in Table 1. The highest diffusion values are observed in the spinal cord of infants ${ }^{37}$ and the lowest in adults with an age range of 30 to 50 years. ${ }^{38,39}$ In subjects older than 50 years, the spinal cord diffusion appears to be higher, but on average not as high as in children. The studies cited in Table 1 do not include FA values for infants and children, but report anisotropy indices that indicate lower FA values during development. The developmental and age dependence of mean diffusivity and FA is well known and has also been observed in the brain. ${ }^{40,41}$ However, not necessarily all of these changes may be present in the spinal cord tissue, but may rather be a manifestation of the limited spatial resolution together with CSF partial volume effects and age-related changes in spinal cord geometry. Indeed, CSF contamination may cause an overestimation of diffusion values and an underestimation of FA values. Such contamination most likely occurs in sagittal

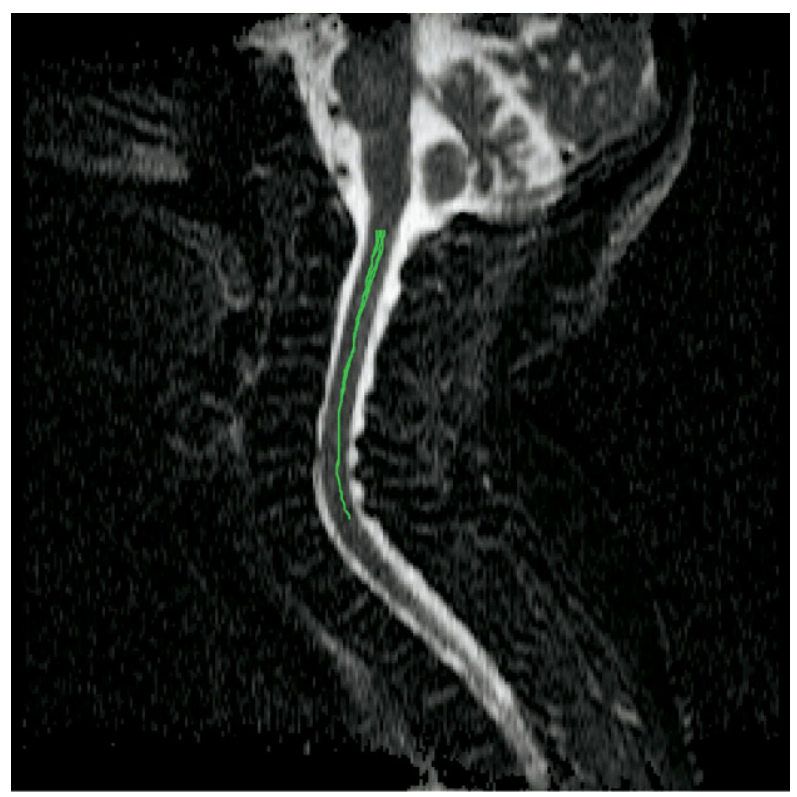

FIG. 3. Sagittal line scan trace diffusivity map of a healthy $72-$ year-old female patient with an almost kyphotic back. Fiber tracking along the eigenvectors with the largest diffusivity, visualized with an overlay of green lines, follows exactly the bent fiber structure of the spinal cord. 


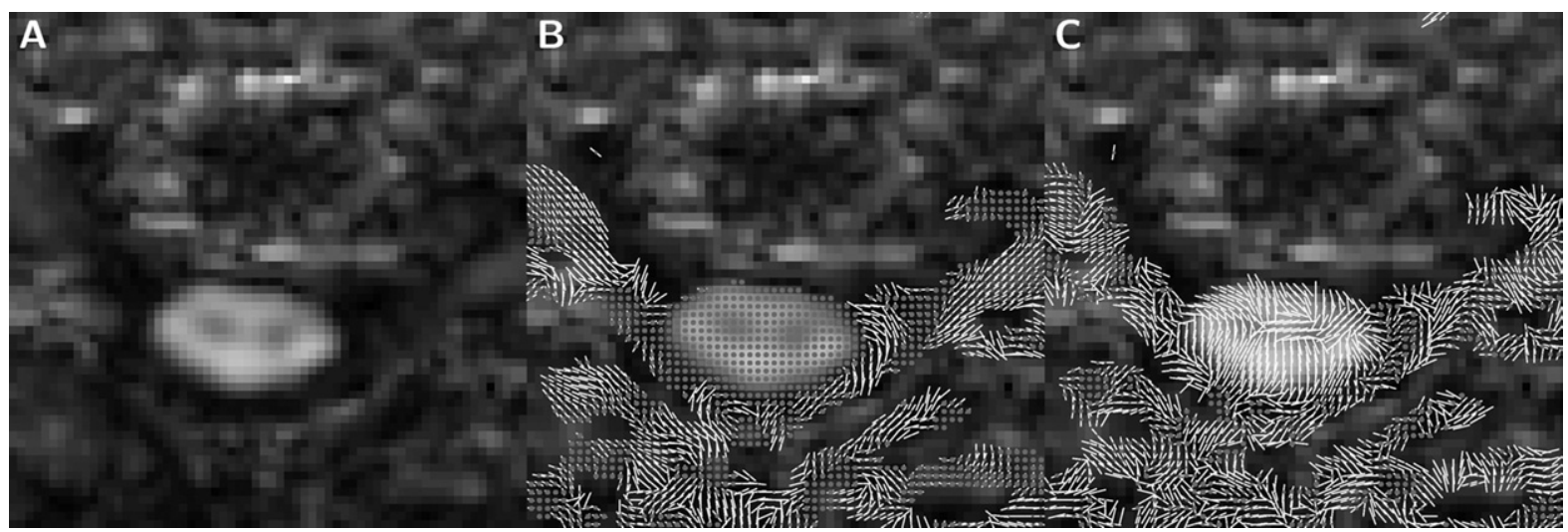

FIG. 4. Axial line scan diffusion tensor image data of a healthy 35-year-old female volunteer. The scan was performed at the C6 level of the cervical spinal cord with a spatial resolution of $1.25 \times 1.25 \times 4 \mathrm{~mm}$. To reliably map the eigenvector directions, magnetic resonance signals were acquired 20 times and were averaged. A: Fractional anisotropy (FA) map showing an area of $40 \times 40 \mathrm{~mm}$. In the center of the image, the spinal cord can be seen surrounded by CSF, which appears dark due to low anisotropy. Within the spinal cord cross-section, the dark central zone of low anisotropy corresponds to the central gray matter, whereas the surrounding bright zone of high anisotropy corresponds to the peripheral white matter. B: Same FA map as in (A) overlaid with the first eigenvector directions. The out-of-plane component is represented by gray dots of different sizes and the in-plane component by white lines. Eigenvectors are only displayed for pixels, where the intensity in the diffusion-weighted image exceeds a predefined threshold. In agreement with known anatomy, within the spinal cord the first eigenvector is largely orthogonal to the axial plane. Right and left to the spinal cord, the eigenvectors clearly follow the direction of the spine nerve roots. C: Same FA map as in (A) overlaid with the second eigenvector directions. The second eigenvectors are arranged in a centrifugal pattern, which was also observed in the spinal cord specimen shown in Figure 2.

sections, when the voxel geometry is anisotropic. The sagittal and axial plane diffusivity values listed in Table 1 show no major difference, which indicates a negligible CSF signal contamination. The remarkable difference between FA measurements obtained with signal averaging and FA measurements obtained without it underlines the importance of an adequate signal-to-noise ratio, particularly in areas of low anisotropy, such as spinal cord gray matter or cerebral cortex.

\section{DIRECTIONALLY DEPENDENT DIFFUSION AND SPINAL CORD TISSUE ARCHITECTURE}

The assessment of the diffusion eigenvector for each image voxel allows for accurate mapping of the axon directions. With the aid of diffusion tensor tractography, pathways of nerve fibers can be reconstructed over extended lengths of the spinal cord. ${ }^{17,27}$ By following repetitively in small steps along spatially interpolated directions of maximum diffusion, a contiguous path that passes through initial seed positions is defined. An example of spinal cord fiber tracking is presented in Figure 3. Thus, the departure from the basic assessment of the anisotropy of each diffusion ellipsoid to the more elaborate analysis of neighbor relationships among diffusion ellipsoids opens the possibility for assessing in vivo axonal fiber connectivity and functional links between the spinal cord and brain regions. ${ }^{17}$

An analysis of the second eigenvector directions, derived from highly signal-averaged diffusion tensor scans, reveals an orderly arrangement (FIG. 4). On axial sections, the second eigenvectors consistently point from the central gray matter zone to the peripheral white matter zone. A detailed comparison of the second eigenvectors with histological findings revealed an excellent agreement between second eigenvector and collateral fiber orientation. ${ }^{42}$ Thus, the presence of secondary fibers that run at an angle to the primary fiber direction increases the diffusion restriction

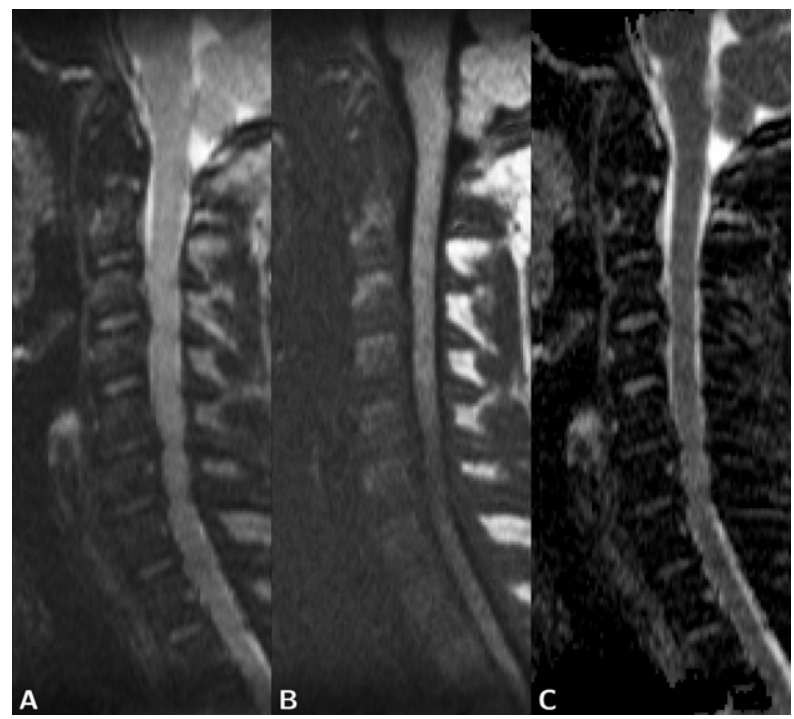

FIG. 5. Sagittal line scan diffusion tensor image data of the cervical spinal cord in a 52-year-old male patient with spondylosis. A: T2-weighted image. B: Diffusion-weighted image reconstructed from image data along six diffusion encoding directions and extrapolated to $b=1000 \mathrm{~s} / \mathrm{mm}^{2}$. C: Trace diffusivity map. Tissue water diffusion at the C5-C6 level (where a narrowing of the spinal canal is evident) is elevated to $0.88 \mu \mathrm{m}^{2} / \mathrm{ms}$. Tissue water diffusion amounted to $0.72 \mu \mathrm{m}^{2} / \mathrm{ms}$ below and 0.78 $\mu \mathrm{m}^{2} / \mathrm{ms}$ above the narrow spinal canal segment. An artifactual elevation of the measured diffusivity within the narrow spinal canal zone by partial CSF volumes is unlikely, because very little CSF surrounds the compressed cord. 


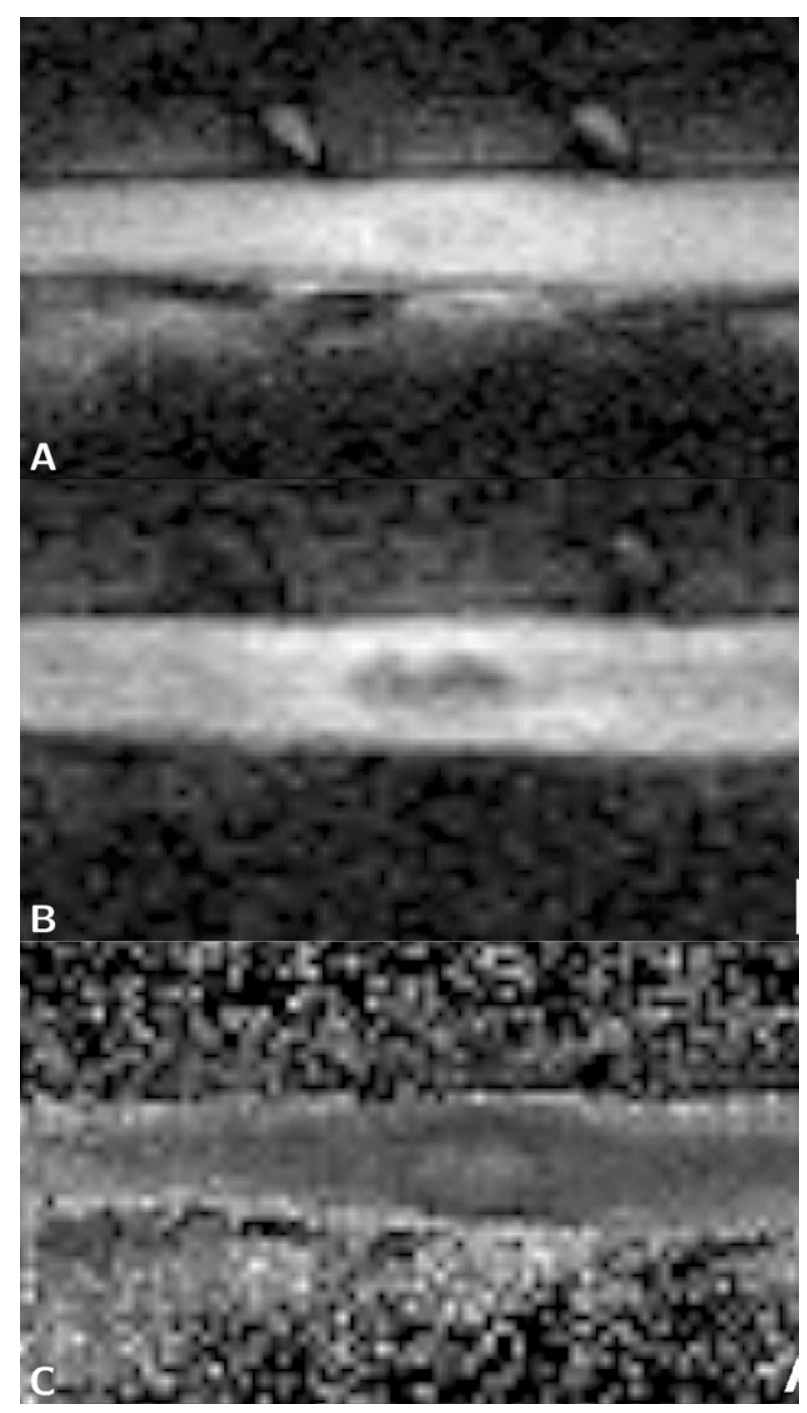

FIG. 6. Sagittal diffusion tensor image data obtained in a rat contusion injury model. ${ }^{50}$ (Images courtesy of Stanislaw Kwiecinski and Tomasz Banasik, Institute of Nuclear Physics PAN, Krakow, Poland.) A: T2-weighted image. B: Diffusion-weighted image. C: Trace diffusivity map. The acute lesion is visible on all three types of images, but particularly distinct on the diffusionweighted image and the diffusivity map.

along the primary fiber direction and reduces the diffusion restriction along the secondary fiber direction. The third eigenvector, with the lowest diffusivity, points along the direction of most severe diffusion restriction (i.e., orthogonal to the primary and secondary fiber direction). The fiber architecture in the spinal cord is special insofar as the secondary collateral nerve fibers run at a right angle to the primary longitudinal fibers, and therefore the simplified diffusion tensor model with three orthogonal eigenvector directions is perfectly adequate. In the presence of nonorthogonal fiber crossings, fiber architecture can be assessed by measuring tissue diffusion properties along a large number of directions. ${ }^{43}$

\section{DIFFUSION IMAGING OF THE DISEASED SPINAL CORD}

Diffusion imaging can be useful to document pathologic changes of the spinal cord. One of the most common changes is the elevation of the tissue diffusion as an early indicator of tissue damage in the compressed spinal cord section of spondylosis patients (FIG. 5). ${ }^{14,38}$ Spondylotic myelopathy with irreversible tissue damage is usually associated with abnormally low diffusion anisotropy. In multiple sclerosis patients, diffusivity and anisotropy change in a similar fashion over the entire spinal cord. ${ }^{44}$ In patients with tumors, assessment of tissue water diffusion can aid in the definitive lesion characterization. ${ }^{45}$ As in the brain, the immediate reduction of diffusivity after ischemic damage is a valuable indicator of infarction. ${ }^{46}$

The phenomenon of diffusion anisotropy is of particular interest to studies that evaluate the local structural integrity of the spinal cord. Based on geometry and the degree of anisotropy loss, fiber tract alterations (such as dislocation, swelling, infiltration, and disruption) can be documented. Particularly in traumatic spinal cord injury, diffusion tensor imaging in conjunction with fiber tracking can be a valuable tool to assess spinal cord damage. ${ }^{47}$ Of great interest for therapy research is that spinal cord MR diffusion tensor imaging can be used for noninvasive serial documentation of spinal cord injury in animals, such as rats (FIG. 6). ${ }^{48-50}$ With high-field MR systems and implantable radio-frequency coils, high-resolution diffusion tensor imaging can even be performed in the spinal cord of mice. ${ }^{51}$ Thus, diffusion tensor imaging, pending further refinements in image quality and robustness, may become an important routine tool in therapy research for spinal cord trauma patients.

Acknowledgments: This work was supported by grants from the National Institute of Neurological Disorders and Stroke (NIH R01 NS39335) and the National Institute for Biomedical Imaging (NIH R01 EB006867-05A2). Rat spinal cord images are courtesy of Stanislaw Kwiecinski from the Institute of Nuclear Physics PAN, Krakow, Poland.

\section{REFERENCES}

1. Papadakis NG, Xing D, Houston GC, et al. A study of rotationally invariant and symmetric indices of diffusion anisotropy. Magn Reson Imaging 1999; 17:881-892.

2. Takahashi M, Hackney D, Zhang G, et al. Magnetic resonance microimaging of intraaxonal water diffusion in live excised lamprey spinal cord. Proc Natl Acad Sci USA 2002;99:16192-16196.

3. Beaulieu C. The basis of anisotropic water diffusion in the nervous system - a technical review. NMR Biomed 2002;15:435-455.

4. Basser P, Mattiello J, Le Bihan D. MR diffusion tensor spectroscopy and imaging. Biophys J 1994;66:259-267.

5. Hsu EW, Muzikant AL, Matulevicius SA, Penland RC, Henriquez CS. Magnetic resonance myocardial fiber-orientation mapping with direct histological correlation. Am J Physiol 1998;274: H1627-H1634.

6. Lin CP, Tseng WY, Cheng HC, Chen JH. Validation of diffusion 
tensor magnetic resonance axonal fiber imaging with registered manganese-enhanced optic tracts. Neuroimage 2001;14:1035-1047.

7. Hopkins JA, Wehrli FW. Magnetic susceptibility measurement of insoluble solids by NMR: Magnetic susceptibility of bone. Magn Reson Med 1997;37:494-500.

8. Turner R, Le Bihan D, Maier J, Vavrek R, Hedges LK, Pekar J. Echo-planar imaging of intravoxel incoherent motions. Radiology 1990;177:407-414.

9. Summers P, Staempfli P, Jaermann T, Kwiecinski S, Kollias S. A preliminary study of the effects of trigger timing on diffusion tensor imaging of the human spinal cord. AJNR Am J Neuroradiol 2006;27:1952-1961.

10. Clark CA, Barker GJ, Tofts PS. Magnetic resonance diffusion imaging of the human cervical spinal cord in vivo. Magn Reson Med 1999;41:1269-1273.

11. Bammer R, Fazekas F, Augustin M, et al. Diffusion-weighted MR imaging of the spinal cord. AJNR Am J Neuroradiol 2000;21:587591.

12. Ries M, Jones RA, Dousset V, Moonen CT. Diffusion tensor MRI of the spinal cord. Magn Reson Med 2000;44:884-892.

13. Holder CA, Muthupillai R, Mukundan SJ, Eastwood JD, Hudgins P. Diffusion-weighted MR imaging of the normal human spinal cord in vivo. AJNR Am J Neuroradiol 2000;21:1799-1806.

14. Demir A, Ries M, Moonen CT, et al. Diffusion-weighted MR imaging with apparent diffusion coefficient and apparent diffusion tensor maps in cervical spondylotic myelopathy. Radiology 2003; 229:37-43.

15. Zhang J, Huan Y, Qian Y, Sun L, Ge Y. Multishot diffusionweighted imaging features in spinal cord infarction. J Spinal Disord Tech 2005;18:277-282.

16. Nagayoshi K, Kimura S, Ochi M, et al. Diffusion-weighted echo planar imaging of the normal human cervical spinal cord. J Comput Assist Tomogr 2000;24:482-485.

17. Voss HU, Watts R, Ulug AM, Ballon D. Fiber tracking in cervical spine and inferior brain regions with reversed gradient diffusion tensor imaging. Magn Reson Imaging 2006;24:231-239.

18. Le Roux P, Darquie A, Carlier PG, Clark CA. Feasibility study of non Carr Purcell Meiboom Gill single shot fast spin echo in spinal cord diffusion imaging. MAGMA 2002;14:243-247.

19. Tsuchiya K, Katase S, Fujikawa A, Hachiya J, Kanazawa H, Yodo K. Diffusion-weighted MRI of the cervical spinal cord using a single-shot fast spin-echo technique: findings in normal subjects and in myelomalacia. Neuroradiology 2003;45:90-94.

20. Bammer R, Augustin M, Prokesch R, Stollberger R, Fazekas F. Diffusion-weighted imaging of the spinal cord: interleaved echoplanar imaging is superior to fast spin-echo. J Magn Reson Imaging 2002;15:364-373.

21. Gudbjartsson H, Maier SE, Mulkern RV, Morocz IA, Patz S, Jolesz FA. Line scan diffusion imaging. Magn Reson Med 1996; 36:509-519.

22. Maier SE, Gudbjartsson H, Patz S, et al. Line scan diffusion imaging: characterization in healthy subjects and stroke patients. AJR Am J Roentgen 1998;17:85-93.

23. Robertson RL, Maier SE, Mulkern RV, Vajapayam S, Robson CD, Barnes PD. MR line-scan diffusion imaging of the spinal cord in children. AJNR Am J Neuroradiol 2000;21:1344-1348.

24. Hori M, Okubo T, Aoki S, et al. Line scan diffusion weighted imaging (LSDI) on 0.2 Tesla MRI of the normal cervical cord in vivo: preliminary study. Nippon Igaku Hoshasen Gakkai Zasshi 2002;62:221-223.

25. Mamata H, Jolesz FA, Maier SE. Characterization of central nervous system structures by magnetic resonance diffusion anisotropy. Neurochem Int 2004;45:553-560.

26. Maier SE. Slab scan diffusion imaging. Magn Reson Med 2001; 46:1136-1143.

27. Wheeler-Kingshott CA, Hickman SJ, Parker GJ, et al. Investigating cervical spinal cord structure using axial diffusion tensor imaging. Neuroimage 2002;16:93-102.

28. Wilm BJ, Svensson J, Henning A, Pruessmann KP, Boesiger P, Kollias SS. Reduced field-of-view MRI using outer volume suppression for spinal cord diffusion imaging. Magn Reson Med 2007; $57: 625-630$
29. Cercignani M, Horsfield MA, Agosta F, Filippi M. Sensitivityencoded diffusion tensor MR imaging of the cervical cord. AJNR Am J Neuroradiol 2003;24:1254-1256.

30. Tsuchiya K, Fujikawa A, Suzuki Y. Diffusion tractography of the cervical spinal cord by using parallel imaging. AJNR Am J Neuroradiol 2005;26:398-400.

31. Falconer JC, Narayana PA. Cerebrospinal fluid-suppressed highresolution diffusion imaging of human brain. Magn Reson Med 1997;37:119-123.

32. Bastin M, Armitage P, Marshall I. A theoretical study of the effect of experimental noise on the measurement of anisotropy in diffusion imaging. Magn Reson Imaging 1998;16:773-785.

33. Dietrich O, Heiland S, Sartor K. Noise correction for the exact determination of apparent diffusion coefficients at low SNR. Magn Reson Med 2001;45:448-453.

34. Hakyemez B, Aksoy U, Yildiz H, Ergin N. Intracranial epidermoid cysts: diffusion-weighted, FLAIR and conventional MR findings. Eur J Radiol 2005;54:214-220.

35. Schwartz ED, Cooper ET, Fan Y, et al. MRI diffusion coefficients in spinal cord correlate with axon morphometry. Neuroreport 2005; 16:73-76.

36. Golabchi F, Brooks D, Hoge W, Mamata H, Maier SE. Comparison of MR diffusion anisotropy with axon density. In: Book of Abstracts, Fourteenth Annual Meeting, Seattle. Society of Magnetic Resonance, Berkeley California, 2006.

37. Murphy BP, Zientara GP, Huppi PS, et al. Line scan diffusion tensor MRI of the cervical spinal cord in preterm infants. J Magn Reson Imaging 2001;13:949-953.

38. Mamata H, Jolesz FA, Maier SE. Apparent diffusion coefficient and fractional anisotropy in spinal cord: age and cervical-spondylosis related changes. J Magn Reson Imaging 2005;22:38-43.

39. Maier SE, Mamata H. Diffusion tensor imaging of the spinal cord. Ann NY Acad Sci 2005;1064:50-60.

40. Huppi PS, Maier SE, Peled SS, et al. Microstructural development of the human newborn cerebral white matter assessed in vivo by diffusion tensor MRI. Pediatr Res 1998;44:584-590.

41. Pfefferbaum A, Sullivan EV, Hedehus M, Lim KO, Adalsteinsson E, Moseley M. Age-related decline in brain white matter anisotropy measured with spatially corrected echo-planar diffusion tensor imaging. Magn Reson Med 2000;44:259-268.

42. Mulkern RV, Davis PE, Haker SJ, et al. Complementary aspects of diffusion imaging and fMRI; I: structure and function. Magn Reson Imaging 2006;24:463-474.

43. Tuch DS, Reese TG, Wiegell MR, Makris N, Belliveau JW, Wedeen VJ. High angular resolution diffusion imaging reveals intravoxel white matter fiber heterogeneity. Magn Reson Med 2002;48:577-582.

44. Valsasina P, Rocca MA, Agosta F, et al. Mean diffusivity and fractional anisotropy histogram analysis of the cervical cord in MS patients. Neuroimage 2005;26:822-828.

45. Teksam M, Casey SO, Michel E, Benson M, Truwit CL. Intraspinal epidermoid cyst: diffusion-weighted MRI. Neuroradiology 2001;43:572-574.

46. Shinoyama M, Takahashi T, Shimizu H, Tominaga T, Suzuki M. Spinal cord infarction demonstrated by diffusion-weighted magnetic resonance imaging. J Clin Neurosci 2005;12:466-468.

47. Facon D, Ozanne A, Fillard P, Lepeintre JF, Tournoux-Facon C, Ducreux D. MR diffusion tensor imaging and fiber tracking in spinal cord compression. AJNR Am J Neuroradiol 2005;26:1587-1594.

48. Bilgen M. A new device for experimental modeling of central nervous system injuries. Neurorehabil Neural Repair 2005; 19:219-226.

49. Deo AA, Grill RJ, Hasan KM, Narayana PA. In vivo serial diffusion tensor imaging of experimental spinal cord injury. J Neurosci Res 2006;83:801-810.

50. Banasik T, Jasiński A, Pilc A, Majcher K, Brzegowy P. Application of magnetic resonance diffusion anisotropy imaging for the assessment of neuroprotecting effects of MPEP, a selective mGluR5 antagonist, on the rat spinal cord injury in vivo. Pharmacol Rep 2005;57:861-866.

51. Bilgen M. Magnetic resonance microscopy of spinal cord injury in mouse using a miniaturized implantable RF coil. J Neurosci Methods 2007;159:93-97. 